The Journal of $\mathbf{N}_{\text {onlinear }} \mathbf{S}$ ciences and Applications http://www.tjnsa.com

\title{
SEVERAL DISCRETE INEQUALITIES FOR CONVEX FUNCTIONS
}

\author{
XINKUAN CHAI ${ }^{1}$, YONGGANG ZHAO ${ }^{2}$ AND HONGXIA DU ${ }^{3 *}$
}

ABstract. In this paper, we establish some interesting discrete inequalities involving convex functions and pose an open problem.

\section{INTRODUCTION}

The following problem was posed by Qi in his article [13]: "Under what condition does the inequality

$$
\int_{a}^{b}[f(x)]^{t} d x \geq\left(\int_{a}^{b} f(x) d x\right)^{t-1}
$$

hold for $t>1$ ?".

There are numerous answers and extension results to this open problem [1, 2, $3,4,5,6,7,8,11,12,14,15,16$. These results were obtained by different approaches, such as, e.g. Jensen's inequality, the convexity method [16]; functional inequalities in abstract spaces [1, 2]; probability measures view [4, 7]; Hölder inequality and its reversed variants [2, 12]; analytical methods [11, 15]; Cauchy's mean value theorem [3, 14].

In [9], the authors introduced the following discrete version of (1.1) as follows, "Under what condition does the inequality

$$
\sum_{i=1}^{n} x_{i}^{\alpha} a_{i} \geq\left(\sum_{i=1}^{n} x_{i} a_{i}\right)^{\beta}
$$

Date: Received: 18 March 2010.

* Corresponding author

(c) 2010 N.A.G.

2000 Mathematics Subject Classification. Primary 26D15.

Key words and phrases. Qi-type inequality, discrete inequality, convex functions. 
hold for $\alpha, \beta>0$ ?". (For the infinite series, the same method in the above finite series can be discussed.) Very recently, some similar discrete inequalities were developed (for instance, the reference [10]). In the paper, based on the results in [5], we will establish some discrete type inequalities and pose an open problem.

\section{MAIn RESUlts}

Before starting the results for convex function, we firstly show the following results.

Theorem 2.1. Let $\left\{x_{i}, i=1, \ldots, n\right\},\left\{y_{i}, i=1, \ldots, n\right\}$ be two sequences of nonnegative real numbers such that $x_{i} \leq y_{i}$ for all $1 \leq i \leq n$,

Then we have

$$
\frac{x_{1}}{y_{1}} \geq \frac{x_{2}}{y_{2}} \geq \cdots \geq \frac{x_{n}}{y_{n}} \text { and } x_{1} \leq x_{2} \leq \cdots \leq x_{n} .
$$

for all $p \geq 1$. If

$$
\frac{\sum_{i=1}^{n} x_{i}}{\sum_{i=1}^{n} y_{i}} \geq \frac{\sum_{i=1}^{n} x_{i}^{p}}{\sum_{i=1}^{n} y_{i}^{p}}
$$

$$
\frac{x_{1}}{y_{1}} \leq \frac{x_{2}}{y_{2}} \leq \cdots \leq \frac{x_{n}}{y_{n}} \quad \text { and } \quad x_{i} \geq y_{i}
$$

for all $1 \leq i \leq n$, then the inequality in (2.1) reverses.

Proof. Let $z_{i}=x_{i}^{p-1}$, then $z_{1} \leq z_{2} \leq \cdots \leq z_{n}$ by $p \geq 1$. From the assumptions of Theorem 2.1, we have

$$
\left(z_{i}-z_{j}\right)\left(\frac{x_{j}}{y_{j}}-\frac{x_{i}}{y_{i}}\right) \geq 0, \text { for all } 1 \leq i, j \leq n .
$$

Firstly we need to prove

This is to say

$$
\frac{\sum_{i=1}^{n} x_{i}}{\sum_{i=1}^{n} y_{i}} \geq \frac{\sum_{i=1}^{n} x_{i} z_{i}}{\sum_{i=1}^{n} y_{i} z_{i}}
$$

$$
\sum_{i=1}^{n} x_{i} \sum_{i=1}^{n} y_{i} z_{i} \geq \sum_{i=1}^{n} y_{i} \sum_{i=1}^{n} x_{i} z_{i}
$$

which is equivalent to

$$
D:=\sum_{i=1}^{n} \sum_{j=1}^{n} z_{j}\left(x_{i} y_{j}-y_{i} x_{j}\right) \geq 0 .
$$

Noting

$$
D=\sum_{i=1}^{n} \sum_{j=1}^{n} z_{i}\left(x_{j} y_{i}-y_{j} x_{i}\right)
$$

then we have

$$
\begin{aligned}
2 D & =\sum_{i=1}^{n} \sum_{j=1}^{n}\left(z_{i}-z_{j}\right)\left(x_{j} y_{i}-y_{j} x_{i}\right) \\
& =\sum_{i=1}^{n} \sum_{j=1}^{n} y_{i} y_{j}\left(z_{i}-z_{j}\right)\left(\frac{x_{j}}{y_{j}}-\frac{y_{i}}{x_{i}}\right)
\end{aligned}
$$


which yields the inequality (2.3) by the condition (2.2). Since $x_{i} \leq y_{i}$ for all $1 \leq i \leq n$, then

$$
\begin{aligned}
\frac{\sum_{i=1}^{n} x_{i}}{\sum_{i=1}^{n} y_{i}} & \geq \frac{\sum_{i=1}^{n} x_{i} z_{i}}{\sum_{i=1}^{n} y_{i} z_{i}} \\
& =\frac{\sum_{i=1}^{n} x_{i}^{p}}{\sum_{i=1}^{n} y_{i} x_{i}^{p-1}} \geq \frac{\sum_{i=1}^{n} x_{i}^{p}}{\sum_{i=1}^{n} y_{i}^{p}}
\end{aligned}
$$

which is the first result. The proof of the other result is similar to (2.1).

Next, we give some inequalities involving convex function.

Theorem 2.2. Let $\left\{x_{i}, i=1, \ldots, n\right\},\left\{y_{i}, i=1, \ldots, n\right\}$ and be two sequences of nonnegative real numbers such that $x_{i} \leq y_{i}$ for all $1 \leq i \leq n$,

$$
\frac{x_{1}}{y_{1}} \geq \frac{x_{2}}{y_{2}} \geq \cdots \geq \frac{x_{n}}{y_{n}} \text { and } x_{1} \leq x_{2} \leq \cdots \leq x_{n} .
$$

Assume that $\phi(x)$ is a convex function with $\phi(0)=0$. Then we have

$$
\frac{\sum_{i=1}^{n} x_{i}}{\sum_{i=1}^{n} y_{i}} \geq \frac{\sum_{i=1}^{n} \phi\left(x_{i}\right)}{\sum_{i=1}^{n} \phi\left(y_{i}\right)} .
$$

Proof. Since $\phi(x)$ is convex with $\phi(0)=0$, then $\frac{\phi(x)}{x}$ is increasing. Hence from $x_{i} \leq y_{i}$ for all $1 \leq i \leq n$, we have

$$
\frac{\phi\left(x_{i}\right)}{x_{i}} \leq \frac{\phi\left(y_{i}\right)}{y_{i}}, \text { for all } 1 \leq i \leq n .
$$

Let $g(x)=\frac{\phi(x)}{x}$, then $g(x)$ is also increasing. So we have

$$
\begin{aligned}
\frac{\sum_{i=1}^{n} \phi\left(x_{i}\right)}{\sum_{i=1}^{n} \phi\left(y_{i}\right)} & =\frac{\sum_{i=1}^{n} x_{i} g\left(x_{i}\right)}{\sum_{i=1}^{n} y_{i} g\left(y_{i}\right)} \\
& \leq \frac{\sum_{i=1}^{n} x_{i} g\left(x_{i}\right)}{\sum_{i=1}^{n} y_{i} g\left(x_{i}\right)} \leq \frac{\sum_{i=1}^{n} x_{i}}{\sum_{i=1}^{n} y_{i}} .
\end{aligned}
$$

Here the last inequality stems from the similar proof of Theorem 2.1.

Theorem 2.3. Let $\left\{x_{i}, i=1, \ldots, n\right\},\left\{y_{i}, i=1, \ldots, n\right\}$ and $\left\{z_{i}, i=1, \ldots, n\right\}$ be three sequences of nonnegative real numbers such that $x_{i} \leq y_{i}$ for all $1 \leq i \leq n$,

$$
\frac{x_{1}}{y_{1}} \geq \frac{x_{2}}{y_{2}} \geq \cdots \geq \frac{x_{n}}{y_{n}}, x_{1} \leq x_{2} \leq \cdots \leq x_{n} \text { and } z_{1} \leq z_{2} \leq \cdots \leq z_{n} .
$$

Assume that $\phi(x)$ is a convex function with $\phi(0)=0$. Then we have

$$
\frac{\sum_{i=1}^{n} x_{i}}{\sum_{i=1}^{n} y_{i}} \geq \frac{\sum_{i=1}^{n} \phi\left(x_{i}\right) z_{i}}{\sum_{i=1}^{n} \phi\left(y_{i}\right) z_{i}} .
$$

Proof. The proof is similar to Theorem 2.3. We have

$$
\begin{aligned}
\frac{\sum_{i=1}^{n} \phi\left(x_{i}\right) z_{i}}{\sum_{i=1}^{n} \phi\left(y_{i}\right) z_{i}} & =\frac{\sum_{i=1}^{n} \frac{\phi\left(x_{i}\right)}{x_{i}} x_{i} z_{i}}{\sum_{i=1}^{n} \frac{\phi\left(y_{i}\right)}{y_{i}} y_{i} z_{i}} \\
& \leq \frac{\sum_{i=1}^{n} \frac{\phi\left(x_{i}\right)}{x_{i}} x_{i} z_{i}}{\sum_{i=1}^{n} \frac{\phi\left(x_{i}\right)}{x_{i}} y_{i} z_{i}} \leq \frac{\sum_{i=1}^{n} x_{i}}{\sum_{i=1}^{n} y_{i}} .
\end{aligned}
$$


At last, we give an open problem as follows.

Open Problem 1. Suppose that $\phi(x)$ is a convex function with $\phi(0)=0$. Under what conditions does the inequality

$$
\frac{\sum_{i=1}^{n} x_{i}}{\sum_{i=1}^{n} y_{i}} \geq \frac{\left(\sum_{i=1}^{n} \phi\left(x_{i}\right) z_{i}\right)^{\delta}}{\left(\sum_{i=1}^{n} \phi\left(y_{i}\right) z_{i}\right)^{\lambda}}
$$

hold for $\delta, \lambda$ ?

\section{REFERENCES}

[1] M. Akkouchi, On an integral inequality of Feng Qi, Divulg. Mat., 13 (2005), 11-19.

[2] L. Bougoffa, Notes on Qi type integral inequalities, J. Inequal. Pure and Appl. Math., 4 (2003), Art. 77.

[3] Y. Chen and J. Kimball, Note on an open problem of Feng Qi, J. Inequal. Pure and Appl. Math., 7 (2006), Art. 4.

[4] V. Csiszár and T. F. Mòri, The convexity method of proving moment-type inequalities, Statist. Probab. Lett., 66 (2004), 303-313.

[5] W. J. Liu, Q. A. Ngô and V. N. Huy, Several interesting integral inequalities, J. Math. Inequal., 3 (2009), 201-212.

[6] S. Mazouzi and F. Qi, On an open problem regarding an integral inequality, J. Inequal. Pure and Appl. Math., 4 (2003), Art. 31.

[7] Y. Miao, Further development of Qi-type integral inequality, J. Inequal. Pure and Appl. Math., 7 (2006), Art. 144.

[8] I. Miao and J. F. Li, Further development of an open problem, J. Inequal. Pure and Appl. Math., 9 (2008), Art. 108.

[9] I. Miao and J. F. Liu, Discrete results of Qi-type inequality, Bull. Korean Math. Soc., 46 (2009), 125-134.

[10] I. Miao and F. Qi, A discrete version of an open problem and several answers, J. Inequal. Pure and Appl. Math., 10 (2009), Art. 49.

[11] J. Pečarić and T. Pejković, Note on Feng Qi's integral inequality, J. Inequal. Pure and Appl. Math., 5 (2004), Art. 51.

[12] T. K. Pogány, On an open problem of F. Qi, J. Inequal. Pure and Appl. Math., 3 (2002), Art. 54.

[13] F. Qi, Several integral inequalities, J. Inequal. Pure and Appl. Math., 1 (2000), Art. 19.

[14] F. Qi, A. J. Li, W. Z. Zhao, D. W. Niu and J. Cao, Extensions of several integral inequalities, JIPAM. J. Inequal. Pure and Appl. Math., 7 (2006), Art. 107.

[15] N. Towghi, Notes on integral inequalities, RGMIA Res. Rep. Coll., 4 (2001), Art. 10, $277-278$.

[16] K.-W. Yu and F. Qi, A short note on an integral inequality, RGMIA Res. Rep. Coll., 4 (2001), Art. 4, 23-25.

${ }^{1}$ College of Mathematics and Information Science, Henan Normal University, Henan Province, 453007, China.

E-mail address: chaixinkuan@gmail.com

2 College of Mathematics and Information Science, Henan Normal University, Henan Province, 453007, China.

E-mail address: ztyg68@tom.com 
3 College of Mathematics and Information Science, Henan Normal University, Henan Province, 453007, China.

E-mail address: duhongxia24@gmail.com 